\title{
Mensch-Technik-Interaktion im demographischen Wandel
}

\section{Anthropologische Erwägungen zur Gerotechnologie}

Zusammenfassung: Angesichts der steigenden Lebenserwartung, der Auflösung traditioneller Sorgestrukturen sowie des Fachkräftemangels in der professionellen Pflege wird für die Lebensgestaltung im höheren Alter und die pflegerische Versorgung älterer Menschen verstärkt auf technische Lösungen gesetzt. Der Beitrag nähert sich der damit einhergehenden Entwicklung der Mensch-TechnikInteraktion im demographischen Wandel aus dem Blickwinkel der philosophischen Anthropologie. Ziel ist es, die Bedeutung anthropologischer Aspekte und Dimensionen für die Auseinandersetzung mit so genannten Gerotechnologien auszuloten. Dazu gebe ich zunächst einen orientierenden Überblick über das Spektrum und die Entwicklungsperspektiven technischer Assistenzsysteme für ältere Menschen. Im Anschluss wird die Rolle individueller und gesellschaftlicher Altersbilder in der Diskussion und Anwendung dieser Gerotechnologien beleuchtet. Vor diesem Hintergrund werden schließlich einige grundlegende anthropologische Fragen und Desiderate der Mensch-Technik-Interaktion im Alter angesprochen. Sie betreffen insbesondere die Gesichtspunkte der körperlichen Verfasstheit, der zeitlichen Erstreckung und Verlaufsstruktur sowie der relationalen Ausrichtung und Einbettung menschlichen Seins.

\section{Einleitung}

Im antiken Ödipus-Mythos fragt die Sphinx auf der Stadtmauer von Theben die Vorübergehenden nach einem Wesen, das ,am Morgen vierfüßig, am Mittag zweifüßig, am Abend dreifüßig“ (Schwab 1986, 259) sei. Erst Ödipus gelingt es, das Rätsel aufzulösen: Es ist der Mensch, „der am Morgen seines Lebens, solange er ein Kind ist, auf zwei Füßen und zwei Händen kriecht. Ist er stark geworden, geht er am Mittag seines Lebens auf zwei Füßen, am Lebensabend, als Greis, bedarf er der Stütze und nimmt den Stab als dritten Fuß zu Hilfe“ (ebd.).

Es erscheint reizvoll, das Rätsel der Sphinx als mythische Urszene und kulturgeschichtliches Leitmotiv einer anthropologischen Auseinandersetzung mit technischer Assistenz im Alter auszulegen. Es verknüpft die Frage nach dem Menschen sowohl mit dem Vorgang des Alterns und der Stufe des höheren Le- 
bensalters als auch mit der bereits in der griechischen Antike geläufigen Deutung der Technik als Mittel zum Ausgleich natürlicher Unzulänglichkeiten. Das Bemerkenswerte und anthropologisch Kennzeichnende an der Geschichte ist nicht allein, dass der Mensch altert und hinfällig wird, sondern dass er sich dabei in Gestalt des Stabes auf ein - im weitesten Sinne - technisches Hilfsmittel zu stützen vermag.

Freilich hat das Thema in den letzten beiden Jahrzehnten beträchtlich an Aktualität und Konkretion gewonnen. Im Zeichen der steigenden Lebenserwartung, der Auflösung traditioneller familialer Sorgestrukturen sowie des Fachkräftemangels in der professionellen Pflege wird für die Lebensgestaltung im höheren Alter und die pflegerische Versorgung älterer Menschen verstärkt auf technische Lösungen gesetzt (Schmidt/Wahl 2019). Tracking- und Monitoringgeräte erlauben die fortlaufende Überwachung und Kontrolle vitaler Parameter, körperlicher Bewegungsabläufe und alltäglicher Lebensvollzüge. Neue Entwicklungen im Bereich der Robotik stellen den Einsatz assistiver Technologien zur Unterstützung von Aktivitäten des täglichen Lebens oder pflegerischen Arbeitsvorgängen in Aussicht. Umfassende Ambient-Assisted-Living-Anlagen verwandeln das gesamte Wohn- und Lebensumfeld älterer Menschen in eine vernetzte intelligente Umwelt, ein ,Technotop', das ein selbstständiges und bedürfnisgerechtes Leben bis ins hohe Alter ermöglichen soll.

Das Aufkommen dieser „Gerotechnologien“ (Wahl 2016) wirft vielfältige moralische und politische Fragen auf. Die Auseinandersetzung mit ihnen bewegt sich bislang weitgehend auf der Grundlage anderweitig bewährter normativer Grundsätze und Maßstäbe, die auf die neuen technologischen Entwicklungen angewendet werden (Schicktanz/Schweda 2021). Während die Seite der Technik in der Mensch-Technik-Interaktion im Alter so inzwischen bis in Einzelheiten ausgeleuchtet wird, erscheint der Part des (alten) Menschen allerdings noch immer eigentümlich unbestimmt. Das mag nicht zuletzt darauf zurückzuführen sein, dass philosophische wie angewandte Ethik von anthropologischen Voraussetzungen ausgehen, in denen das menschliche Alter(n) kaum grundsätzlich und systematisch mitgedacht wird (Schweda/Coors/Bozzaro 2020; Holm 2013). Stattdessen finden vielfach überkommene und fragwürdige Bilder und Vorstellungen des Alter(n)s bzw. alter Menschen ungeprüft Eingang in die Auseinandersetzung. Verbreitet tritt ein abstraktes Standardsubjekt auf, hinter dem sich bei näherem Zusehen ein selbständiges und unabhängiges Individuum im mittleren Erwachsenenalter verbirgt. Sowohl Kindheit als auch höheres Lebensalter kommen in einer solchen ,adultistischen' Sichtweise von vornherein allenfalls als randständige und abweichende, wenn nicht gar defizitäre Formen des Menschseins in den Blick (Jecker 2020). 
Vor diesem Hintergrund nähert sich der vorliegende Beitrag dem Thema der Mensch-Technik-Interaktion im höheren Lebensalter aus dem Blickwinkel der philosophischen Anthropologie. Ziel ist es, die Bedeutung anthropologischer Aspekte und Dimensionen für die Auseinandersetzung mit der Gerotechnologie auszuloten und in dieser Hinsicht einige grundsätzliche Fragen und theoretische Perspektiven aufzuzeigen. Der Begriff der philosophischen Anthropologie ist dabei weder mit der Zuordnung zu einer bestimmten Schule oder Strömung noch mit dem Anspruch einer feststellenden oder gar abschließenden Bestimmung des (alten) Menschen als solchen verbunden. Er umreißt hier vielmehr eine allgemeine Betrachtungsweise, die auf die Erörterung grundlegender Annahmen des menschlichen Selbst- und Weltverständnisses im Horizont umfassenderer geistesund kulturgeschichtlicher Deutungsmuster ausgerichtet ist (Fischer 2008, 9). Der Beitrag gibt zunächst einen orientierenden Überblick über das Spektrum und die gegenwärtigen Entwicklungsperspektiven technischer Assistenzsysteme für alternde Gesellschaften. Er beleuchtet vor diesem Hintergrund sodann die Rolle individueller und gesellschaftlicher Altersbilder in der Diskussion und Anwendung dieser Gerotechnologien. Im Anschluss werden einige grundlegende anthropologische Fragen und Desiderate der Mensch-Technik-Interaktion im Alter angesprochen. Wie dabei deutlich wird, verdienen insbesondere die Gesichtspunkte der körperlichen Verfasstheit, der zeitlichen Erstreckung und Verlaufsstruktur sowie der relationalen Ausrichtung und Einbettung menschlichen Seins eine eingehendere Auseinandersetzung.

\section{Das Spektrum der Gerotechnologien}

Im Zuge der digitalen Transformation hat sich der Bereich technischer Assistenzsysteme für das Alter(n) in den zurückliegenden 20 Jahren geradezu explosionsartig entwickelt. Aus dem - mutmaßlich hölzernen - Stab, von dem im mythischen Rätsel der Sphinx die Rede war, ist in der Zwischenzeit eine kaum mehr zu überschauende Vielzahl an technischen Verfahren, Gerätschaften und Vorrichtungen geworden, die die selbstständige Lebensführung im höheren Alter unterstützen und die pflegerische Versorgung älterer Menschen erleichtern und verbessern sollen. Das Spektrum reicht von fortgeschrittenen Varianten des herkömmlichen Hausnotrufknopfs über einschlägige Gesundheitsanwendungen auf der Smart Watch bis hin zum humanoiden Pflegeroboter oder der umfassenden Smart Home-Anlage. Hinsichtlich ihres Zwecks und ihrer Funktionsweise lassen sich verschiedene Arten solcher technischen Systeme unterscheiden (Byrne/Collier/O’Hare 2018). 
Tracking- und Monitoringsysteme nutzen Ortungs- und Sensortechnologien, um das alltägliche Leben und die gesundheitliche Verfassung älterer Menschen $\mathrm{zu}$,überwachen' und unerwünschte Ereignisse oder Entwicklungen frühzeitig zu erkennen und gegenzusteuern. Sie können unmittelbar am Körper oder der Kleidung getragen (wearable) oder im Lebensumfeld der Nutzenden (ambient) installiert werden. Dazu gehören etwa Vorrichtungen zur Sturzerkennung mit Tiefenkameras, Infrarotsensoren zur Überwachung der körperlichen Bewegung und Lage einer Person im Raum oder Bodendrucksensoren, die längere Zeiträume der Reglosigkeit erkennen können (Chaudhuri/Thompson/Demiris 2014). Ein weiteres Anwendungsgebiet ist die Unterstützung der räumlichen Orientierung und ,Navigation“ der Nutzenden durch GPS- oder Radartechnologien sowie die Einhegung von ,Weglauftendenzen“ durch Geofencing bei älteren Menschen mit kognitiven Beeinträchtigungen (Ray/Dash/De 2019). Monitoringsysteme lassen sich je nach eingesetzter Sensorik zur Kontrolle von Vitalparametern, physiologischen Funktionen und Verhaltensmustern einsetzen (Stavropoulos et al. 2020). So gibt es Wearables, die kontinuierlich physiologische Parameter wie Puls, Blutdruck und Blutzuckerspiegel messen und bei Abweichung von individualisierten Normwerten einen Alarm auslösen. Darüber hinaus werden Systeme mit ambienter Sensorik eingesetzt, die über die Auswertung von Bewegungsdaten oder Wasser- und Stromverbrauch auch Aktivitäten des täglichen Lebens wie Aufstehen, Körperpflege, Ernährung und Bewegung überwachen und bei Bedarf ein Eingreifen ermöglichen sollen (Pol et al. 2013).

Ein weiteres rasch wachsendes Gebiet bildet die Robotik (s. Giese in diesem Band). Allgemein sind Roboter Automaten, die Sensor-, Prozessor- und Aktuatortechnologien verbinden, um gezielt in die physische Welt einzugreifen. Im gerotechnologischen Kontext umfassen sie sowohl physische Bewegungshilfen als auch Service- und Begleitroboter für spezifische Pflege- oder Haushaltsaufgaben (Maalouf et al. 2018). Als Bewegungshilfen sollen Robotertechnologien genutzt werden, um das Bewegungsvermögen körperlich beeinträchtigter älterer Menschen zu unterstützen, z.B. durch intelligente Gehhilfen oder Greifvorrichtungen. Hierher gehören auch Neuroprothesen und Exoskelette, die über Muskelbewegungen oder Gehirn-Computer-Schnittstellen gesteuert werden und die körperliche Beweglichkeit und Mobilität der Tragenden verbessern können (Cangelosi/Invitto 2017). Einige Robotersysteme sollen eine umfassende physische Unterstützung von Aktivitäten des täglichen Lebens, der Gesundheitsversorgung und der Haushaltsführung bieten. Eine spezielle Teilgruppe stellen sozial unterstützende Roboter dar. Diese Service- und Begleitroboter übernehmen emotionale und soziale Assistenzfunktionen, indem sie zum Beispiel Spiel und Unterhaltung oder Freizeitaktivitäten anregen und unterstützen (Abdi et al. 2018). Schließlich ist auch der Bereich der Pflegerobotik zu nennen, in 
dem Assistenztechnologien insbesondere beschwerliche pflegerische Aufgaben wie das Heben, die Lagerung oder die Mobilisierung älterer Menschen unterstützen oder übernehmen sollen, etwa durch am Pflegebett angebrachte bewegliche Roboterarme (Madara Marasinghe 2016).

Ambient Assisted Living-Systeme (AAL) bilden schließlich umfassende technische Infrastrukturen, die Verfahren des Trackings bzw. Monitorings und Ansätze aus dem Bereich der Robotik im Rahmen von Smart Home-Konzepten kombinieren (Blackman et al. 2016). Sie zielen darauf ab, das gesamte Alltagsleben und Wohnumfeld älterer Menschen unauffällig und bedürfnisgerecht $\mathrm{zu}$ überwachen und zu unterstützen, um ihnen die Aufrechterhaltung eines möglichst unabhängigen Lebens in den eigenen vier Wänden zu erlauben (Meyer/ Mollenkopf 2010). So verbinden AAL-Anlagen etwa Funktionen des Gesundheitsmonitorings, der technischen Unterstützung von körperlicher Beweglichkeit und Aktivitäten des täglichen Lebens, der umfassenden Regulierung von Komfortund Sicherheitsaspekten der Haushaltsführung sowie der Ermöglichung von Unterhaltung, Kommunikation und Teilhabe am gesellschaftlichen Leben (Morris et al. 2013). Beispielsweise lassen sich mit Hilfe von AAL-Technologien zugleich Vitalwerte überwachen und kontrollieren, die Ausführung von Aktivitäten des alltäglichen Lebens wie Kochen, Ankleiden oder Körperpflege durch visuelle oder akustische Aufforderungen, Informationen oder Erinnerungen anleiten und unterstützen und die regelmäßige Reinigung der Wohnung, die flexible Einstellung der Raumtemperatur oder das zuverlässige Schließen von Türen und Fenstern regulieren (Blackman et al. 2016). Dabei fügen sich theoretische Konzepte und technische Ansätze des Ambient Assisted Living in die Vision des ,Aging in Place‘, sicher, unabhängig und selbstbestimmt im angestammten Wohn- und Lebensumfeld alt werden zu können (Callahan Jr. 2019).

Allen diesen Technologien ist gemeinsam, dass sie Mensch-Technik-Interaktion einschließen. Sie lassen sich daher auch als sozio-technische Systeme beschreiben, deren Entwicklung und Implementierung nicht nur technologische Expertise aus Bereichen wie Informatik und Ingenieurswesen, sondern auch psychologisches, soziologisches und - im Falle des Einsatzes im höheren Lebensalter - gerontologisches Fachwissen erfordern (Schulz et al. 2015). Betrachtet man die allgemeine Struktur und Funktionsweise der Mensch-Technik-Interaktion noch eingehender, so lassen sich assistive Technologien für das Alter(n) zusätzlich nach unterschiedlichen Automatisierungsgraden klassifizieren, die Art und Ausmaß der Interaktion bestimmen und die jeweiligen Rollen und Zuständigkeiten der beiden Seiten im sozio-technischen System festlegen (Kaber 2018). Dabei wird das untere Ende der Skala durch weitgehend passive technische Geräte markiert, derer sich die Nutzerinnen und Nutzer wie bloßer Werkzeuge bedienen. In dieser Hinsicht unterscheidet sich etwa ein Badewannenlift, der mit 
Hilfe einer Fernbedienung gesteuert wird, letztlich allenfalls nach Graden technischer Komplexität von dem stützenden Stab aus dem Rätsel der Sphinx. Am anderen Ende der Skala stehen hingegen vollständig automatisierte und in diesem Sinne autonome Systeme, die auf der Grundlage von Verfahren der Künstlichen Intelligenz (KI) und des Maschinellen Lernens (ML) in der Lage sein sollen, ihren Einsatz eigenständig zu steuern, und somit keinerlei aktive Intervention von Seiten des menschlichen Parts mehr erfordern. Zwischen diesen beiden Endpunkten bilden verschiedene Stufen teilautomatisierter Systeme ein breites Mittelfeld. Sie weisen einerseits eine gewisse Fähigkeit zur Selbststeuerung auf, sind aber auf der anderen Seite in unterschiedlichem Maße auf menschliche Entscheidungen und Eingaben angewiesen. Auch wenn jede Form von Techniknutzung interaktive Aspekte haben mag, ist die Interaktion von Mensch und Technik hier also in einem funktional-operativen Sinne dezidiert vorgesehen und erforderlich.

\section{Altersbilder im Kontext der Gerotechnologie}

Indem es die letzte Stufe des menschlichen Lebens durch einen stützenden Stab kennzeichnet, vermittelt das Rätsel der Sphinx ein bis heute geläufiges, geradezu emblematisches Bild des Alter(n)s. Noch auf Verkehrswarnschildern vor zeitgenössischen Senioreneinrichtungen in den USA wird das fortgeschrittene Lebensalter der abgebildeten Fußgängerinnen und Fußgänger unverkennbar durch einen Gehstock signalisiert. Der alte Mensch erscheint vorrangig als körperlich beeinträchtigt und hinfällig. Die im Verlauf der individuellen wie gattungsgeschichtlichen Entwicklung entscheidende Befähigung zum aufrechten Gang scheint ihm wieder abhanden zu kommen. Dabei ist es das technische Hilfsmittel selbst, das seine Nutzerin bzw. seinen Nutzer als im wahrsten Sinne ,unselbstständig‘ ausweist.

Die Bedeutung solcher Altersbilder ist auch im Zusammenhang der Entwicklung, Anwendung und öffentlichen Diskussion aktueller Gerotechnologien keineswegs zu unterschätzen (Durrick et al. 2013). So besteht eine entscheidende Hürde für die Akzeptanz der betreffenden Assistenzsysteme bei ihrer eigentlichen Zielgruppe zeitgenössischer Seniorinnen und Senioren in deren Befürchtung, durch die technische Assistenz eben als ,alt' markiert zu werden (McNeill/Coventry 2015). Dahinter steht ein umfassenderer Wandel individueller und gesellschaftlicher Altersbilder, in dem traditionelle, defizitorientierte Vorstellungen des Alter(n)s an Boden verlieren und verstärkt neue, an den Ressourcen und Potenzialen älterer Menschen ausgerichtete Leitbilder späteren Lebens aufkommen (van Dyk/Lessenich 2009). 
Allgemein sind unter Altersbildern individuelle und gesellschaftliche Vorstellungen des höheren Lebensalters als Zustand, des Alterns als Prozess oder älterer Menschen als Gruppe zu verstehen (Rossow 2012, 11-12). Es kann sich demnach sowohl um psychisch-mentale als auch um soziokulturelle Gegebenheiten handeln, etwa um individuelle Ansichten bzw. Einstellungen oder um kollektive soziale bzw. kulturelle Darstellungen oder Deutungsmuster. Dabei sind Altersbilder allerdings keineswegs bloß im Sinne einfacher Tatsachenbehauptungen über das Alter(n) oder alte Menschen aufzufassen, die sich kurzerhand mit der Wirklichkeit vergleichen und auf diese Weise als wahr oder falsch erweisen ließen. Sie eröffnen vielmehr umfassendere Deutungshorizonte, die auch evaluative und normative Annahmen einschließen.

Als solche können Altersbilder beträchtliche Wirkungen entfalten. Sozialwissenschaftliche Untersuchungen zeigen etwa, dass sie im Wirtschaftsleben und auf dem Arbeitsmarkt eine wichtige Rolle spielen und z. B. einen weitreichenden Einfluss auf Einstellungsentscheidungen und Personalpolitik von Unternehmen haben können (Dordoni/Argentero 2015). Auch im kommerziellen Kundenverkehr und dem öffentlichen Dienstleistungssektor kommt die Macht solcher Altersbilder zur Geltung, etwa in der Art der Ansprache und Beratung (Westberg/Reid/Kopanidis 2019). Schließlich zeigen gerontologische Studien, dass defätistische, defizitorientierte Auffassungen des Alter(n)s auch das Selbstverständnis, das Leistungsvermögen und die Lebensgestaltung älterer Menschen und damit letztlich sogar ihre Gesundheit negativ beeinflussen können (Wurm 2020).

Im Kontext der Gerotechnologie können Altersbilder zunächst in den einschlägigen Diskursen zum Ausdruck kommen. So wird in der akademischen Diskussion, politischen Auseinandersetzung und medialen Berichterstattung über Mensch-Technik-Interaktion im demographischen Wandel immer wieder das erwähnte defizitorientierte Bild des Alter(n)s im Zeichen von Niedergang und Verfall beschworen. Ältere Menschen erscheinen hier vorzugsweise als schwach, unselbstständig und hilfsbedürftig (Vines et al. 2015). Darüber hinaus herrscht die Einschätzung vor, sie wiesen per se eine geringere Technikaffinität und Technikkompetenz auf als jüngere Personen (Künemund 2016). Entsprechend verbreitet ist die Vorstellung, es seien mit Blick auf das Alter besondere Anstrengungen zu unternehmen, um die ,Technik zum Menschen bringen“ (BMBF) zu können (Knowles et al. 2019).

Über ausdrücklichen Zuschreibungen hinaus sind allerdings auch visuelle Repräsentationen des Alter(n)s zu berücksichtigen. So fällt etwa auf, dass mediale Abbildungen des Einsatzes von Pflegerobotik meist überhaupt keine Mitglieder desjenigen Personenkreises zeigen, den diese Technologien vorrangig adressieren. Die eigentliche Zielgruppe der pflegebedürftigen, multimorbiden und gebrechlichen hochbetagten Menschen tritt visuell kaum in Erscheinung. Statt- 
dessen werden häufig Dummies, jüngere Menschen oder Vertreter der so genannten ,jungen', also fitten und aktiven Alten abgebildet. Diese ,optische Verzerrung' mag mit ethischen und rechtlichen Anforderungen des Schutzes vulnerabler Gruppen in frühen Stadien der Technikentwicklung oder der Sorge Herstellender um die Werbewirksamkeit realistischer Abbildungen ihrer Produkte mit Menschen im höchsten Lebensalter zu tun haben. Allerdings fügen sie sich auch in eine verbreitete gesellschaftliche Tendenz, das höchste Alter sozial auszuschließen, institutionell abzukapseln oder kulturell auszublenden und so gleichsam unsichtbar zu machen (Higgs/Gilleard 2014).

Des Weiteren können gesellschaftliche Altersbilder auch schon auf einer grundlegenderen, allen expliziten Aussagegehalten vorgelagerten Ebene die Rahmung, Ausrichtung und Akzentuierung öffentlicher Diskurse über Gerotechnologie beeinflussen. So stellt sich grundsätzlich die Frage, warum die Debatte überhaupt eine derartige Breite und Prominenz erlangt hat. Schließlich haben wir es auch mit Blick auf die Pflege und Betreuung von Säuglingen und Kleinkindern mit ähnlichen Veränderungen und Herausforderungen traditioneller Versorgungsstrukturen zu tun, ohne dass bislang eine vergleichbare öffentliche Diskussion über den Einsatz technischer Assistenzsysteme in diesem Bereich aufgekommen wäre. Hier mögen Bilder des Alter(n)s im Spiel sein, die in beiden Fällen eine unterschiedliche Plausibilität und Akzeptabilität technischer Lösungsansätze nahelegen (Sharkey/Sharkey 2010). Allgemein sind politische und mediale Debatten über technische Assistenz im Alter(n) oft von einer apokalyptischen Hintergrundmetaphorik geprägt, in der sich demographische Krisenszenarien gesellschaftlicher ,Überalterung' mit kulturpessimistischen Zeitdiagnosen sowie professions- und sozialpolitischen Notstandsrhetoriken verbinden (Neven/ Peine 2017). So ist etwa von ,Digitalisierung gegen die Überalterung“ oder ,technischen Innovationen gegen den Pflegenotstand“ die Rede. Das Alter(n) erscheint dabei von vornherein unter negativen Vorzeichen, als individuelles und gesellschaftliches Problem, das mit technischen Mitteln zu bewältigen ist. Individuelle Ressourcen und gesellschaftliche Potenziale alter Menschen bleiben in dieser einseitig defizitorientierten Perspektive weitgehend ausgeblendet.

Neben diesen diskursiven Manifestationen werden Altersbilder auch auf der Ebene der Technisierung des alltäglichen Lebens und der pflegerischen Versorgung selbst unmittelbar praktisch aktualisiert (Wanka und Gallistl 2018). Schon die Entscheidung von Angehörigen oder Pflegeeinrichtungen über den Einsatz technischer Assistenzsysteme ist nicht zuletzt von Vorstellungen von den Bedürfnissen und Bedarfen älterer Menschen bzw. den Anforderungen ihrer pflegerischen Versorgung geprägt. Je nachdem, wie etwa die Problematik der Einsamkeit im Alter aufgefasst wird, dürfte das Urteil über die Eignung eines sozialen Begleitroboters als Lösungsansatz unterschiedlich ausfallen (Pirhonen et 
al. 2020). Auch unterschiedliche Verständnisse der besonderen Aufgaben und Anforderungen der Pflege älterer Menschen legen jeweils andere Einschätzungen der Möglichkeit ihrer technischen Unterstützung nahe (Parviainen/Turja/van Aerschot 2018). Des Weiteren scheint auch die konkrete Nutzung technischer Assistenzsysteme im Alltagsleben sowie der pflegerischen Praxis häufig von Altersbildern geprägt zu sein. So ist aus sozialwissenschaftlichen Studien bekannt, dass im pflegerischen Handeln traditionell so genannte Dependency-SupportScripts zum Tragen kommen, die alten Menschen pauschal Abhängigkeit und Hilfsbedürftigkeit unterstellen und in der Folge selbstständige Verhaltensweisen unterbinden und unselbstständiges Verhalten fördern (Baltes/Wahl 1996). Unterdessen sind freilich eher Vorstellungen einer aktivierenden Pflege in den Vordergrund getreten, die die Ressourcen und Potentiale älterer Menschen zu Selbstständigkeit in den Mittelpunkt rücken. Es bleibt empirisch zu untersuchen und ethisch zu reflektieren, wie technische Assistenzsysteme dabei in die pflegerische Interaktion und die sie leitenden Verhaltensskripte eingebunden werden (Endter 2016).

Schließlich können sich Altersbilder auch auf der Ebene der technischen Artefakte selbst manifestieren und in diesen gewissermaßen materialisieren (Höppner/Urban 2018). Schon die Entscheidung zur Entwicklung eines Pflegeroboters setzt Annahmen über die spezifischen Probleme und Bedarfe älterer Menschen und ihrer pflegerischen Versorgung sowie über die Möglichkeiten technischer Lösungsstrategien voraus. Lange mangelte es in diesem Bereich an systematischer empirischer Forschung zu den Präferenzen der Betroffenen selbst, etwa in Form von nutzerorientierten Bedarfsstudien oder einer partizipativen Zielgruppenbeteiligung, sodass vielfach stereotype Altersbilder in die Technologieentwicklung und damit auch in die konkrete Bauweise technischer Assistenzsysteme eingehen konnten (Mannheim et al. 2019). Der Mechanismus lässt sich schon am Beispiel eines Seniorenhandys verdeutlichen, in dessen bedienungsfreundlichem Design mit übersichtlichem Display und wenigen großen Tasten ein bestimmtes Bild der Fähigkeiten und Schwierigkeiten älterer Nutzender materialisiert ist. Entsprechend können auch in der Struktur und Funktionsweise einer Neuroprothese oder eines Pflegeroboters Annahmen über die besonderen Bedarfe und Anforderungen älterer Menschen verkörpert sein. Das betrifft etwa Vorstellungen dazu, welches Maß an Gesundheit, Funktionalität und Leistungsfähigkeit oder welche Tätigkeiten und Vorhaben im höheren Lebensalter als normal, sinnvoll und wünschenswert gelten können und entsprechend durch technische Assistenz ermöglicht bzw. unterstützt werden sollten (Durick et al. 2013). Allerdings sind keineswegs nur funktionale Aspekte von Bedeutung. Auch in der ästhetischen Gestaltung der Technologie mögen Altersbilder zur Geltung kommen. Mitunter wird dem Roboter ein retrofuturistisches Design ver- 
passt, das die prägenden Technikvorstellungen einer bestimmten Geburtskohorte ansprechen soll. In anderen Fällen wird er als menschliches Gegenüber, eine Art Haustier oder ein kindlich anmutendes Phantasiewesen gestaltet. In alle diese Gestaltungsentscheidungen mögen neben funktionalen Anforderungen auch Annahmen über die Ansprüche und ästhetischen Präferenzen älterer Menschen gegenüber der Technik einfließen (Lee et al. 2016).

\section{Mensch-Technik-Interaktion im Alter als anthropologisches Problem und Desiderat}

Fast hat es den Anschein, als sei die im mythischen Rätsel der Sphinx aufscheinende frühe Einsicht in die entscheidende Bedeutung des Alter(n)s für das Verständnis menschlichen Seins rasch wieder in Vergessenheit geraten (Schweda/ Coors/Bozzaro 2020). Schon in der klassischen griechischen Philosophie zielte die Frage nach dem Menschen jedenfalls vorrangig auf eine allgemeine Begriffsbestimmung ab, in der das menschliche Wesen wie ein in sich abgeschlossenes, gegenständlich vorhandenes Seiendes ein für alle Mal definitorisch erfasst werden sollte. Der Mensch galt fortan etwa als ,zôon politikon', ,animal rationale‘ oder ,homo faber‘. Veränderungen über die Zeit konnten in diesem ,substanzanthropologischen' Bezugsrahmen allenfalls als teleologische Entfaltung oder akzidentelle Oberflächenerscheinung der zu Grunde liegenden Wesensform aufgefasst werden (Trappe 2002). Selbst in der Philosophischen Anthropologie und Existenzphilosophie des beginnenden 20. Jahrhunderts, die sich dezidiert von diesem traditionellen anthropologischen Substanzdenken verabschiedeten, fand zunächst kaum eine systematische Auseinandersetzung mit dem Alter(n) statt (Schweda/Coors/Bozzaro 2020). Erst in jüngerer Zeit wird dem Thema verschiedentlich eine zentrale Bedeutung für die philosophische Verständigung über grundlegende Bedingungen und wesentliche Dimensionen der menschlichen Existenz beigemessen (s. dazu die Übersicht in Schweda 2018).

Das lange vorherrschende, gerontologische Desiderat‘ der Anthropologie scheint allerdings noch in der zeitgenössischen Auseinandersetzung mit der Gerotechnologie nachzuwirken. Schließlich muss jede theoretische oder praktische Beschäftigung mit Mensch-Technik-Interaktion immer schon ein gewisses Verständnis der beiden hier interagierenden Größen voraussetzen und damit letzten Endes unweigerlich auch anthropologische Annahmen in Anspruch nehmen, etwa grundlegende Überzeugungen hinsichtlich der Anlagen und Fähigkeiten des Menschen, der entscheidenden Bedingungen seiner Erhaltung und seines Gedeihens sowie seines Verhältnisses zu technischen Verfahren und Gebilden. So- 
fern das Alter(n) in den entsprechenden anthropologischen Perspektiven nicht mitgedacht wird, eröffnet sich in der Betrachtung der Mensch-Technik-Interaktion im demographischen Wandel gleichsam eine theoretische Leerstelle, in die subjektiv naheliegende oder soziokulturell gängige Altersbilder Eingang finden können. Gerade ,substanzanthropologische‘ Betrachtungsweisen scheinen eine Sicht des Alterns als eines Prozesses der fortschreitenden Zersetzung und Auflösung dessen nahezulegen, was den Menschen eigentlich und wesentlich ausmacht, sodass das höhere Lebensalter als ein Zustand verminderten, defizitären und gleichsam zerfallenden Menschseins erscheint.

Eine eingehendere Auseinandersetzung mit dem Aufkommen der neuen Gerotechnologien wirft vor diesem Hintergrund letztlich die grundsätzliche Frage nach der Bedeutung von Altern und Alter im Zusammenhang menschlichen Seins im Ganzen auf. Dabei kann sich die angesprochene defizitorientierte Sichtweise auf eine lange, ehrwürdige Tradition berufen. Folgt man der schon in der Antike bekannten philosophie- und kulturgeschichtlichen Überlieferung der ,Altersklage', so heißt Alter(n) für den Menschen in erster Linie Abbau, Niedergang und Verfall, ein fortschreitender Verlust wesentlicher menschlicher Eigenschaften, Fähigkeiten und Möglichkeiten (Birkenstock 2008, 21f.). Das äußere Erscheinungsbild verfällt und die körperliche und geistige Kraft, Funktions- und Leistungsfähigkeit lassen nach, es mehren sich Beschwerden, Krankheiten und Gebrechen. Der alternde Mensch wird hilfsbedürftig und abhängig und erscheint in der Folge als eine Last und Bürde für sein näheres Umfeld oder sogar für die Gemeinschaft als Ganze. Das menschliche Leben wird zunehmend mühsam, beschwerlich und neigt sich unaufhaltsam dem Ende. Das hier umrissene Defizitmodell des Alter(n)s hat auch durchaus weitreichende Bedeutung für die grundlegende Einschätzung technischer Assistenz im höheren Lebensalter. Diese erhält in seinem Licht an erster Stelle eine substitutive und kompensatorische Bestimmung: Technik erscheint hier wesentlich als ein Notbehelf, ein Ersatz oder Ausgleich angesichts altersbedingter Funktionsausfälle und Leistungseinbußen menschlichen Seins.

Eine grundlegend andere Sichtweise eröffnet sich dagegen, wenn man der mindestens ebenso alten Tradition des ,Alterslobes' folgt, die die Vorzüge und Segnungen des Älterwerdens und die Tugenden des höheren Lebensalters in den Vordergrund rückt (Birkenstock 2008, 26 f.). Nicht selten wird das Alter(n) hier nach dem Muster jahreszeitlicher Kreisläufe und entsprechender vegetativer Wachstumsprozesse in der agrarischen Welt im Sinne einer Reifung und Vollendung gedeutet und mit Zugewinnen an Lebenserfahrung, Besonnenheit und Gelassenheit in Verbindung gebracht: Es ermöglicht die Befreiung vom ziellosen Ungestüm, den Unsicherheiten und Torheiten der Jugend, insbesondere den noch kaum zu zügelnden Leidenschaften und körperlichen Trieben. Die gesammelte 
Lebenserfahrung vermittelt innere Festigung und abgeklärte Distanz, gelassene Übersicht und Weisheit. In einer solchen Sicht des Alter(n)s im Sinne einer Reifung und Vollendung menschlichen Seins mag auch der Sinn und Zweck von Gerotechnologien in einem grundsätzlich anderen Licht erscheinen. Das gilt zumal, wenn ein dialektischer Bedingungszusammenhang zwischen der mit dem Alter(n) einhergehenden Konfrontation mit den Limitationen, Fragilitäten und Kontingenzen des Menschen auf der einen und dem persönlichen Wachstum auf der anderen Seite hergestellt wird (Kruse 2017). In einer Betrachtungsweise, die das Altern als „Radikalisierung der menschlichen Grundsituation“ und „Werden zu sich selbst“ (Rentsch 2000) begreift, kann der eigentliche Sinn technischer Assistenz jedenfalls nicht vorrangig in der möglichst weitgehenden Vermeidung oder gar Beseitigung alter(n)sbezogener Erfahrungen der Verletzlichkeit, Begrenztheit und Endlichkeit liegen.

Diese im wahrsten Sinne maßgebende Bedeutung anthropologischer Perspektiven auf das Alter(n) für die Auseinandersetzung mit Mensch-Technik-Interaktion im demographischen Wandel tritt vielleicht besonders deutlich mit Blick auf die körperliche Verfasstheit menschlichen Seins zu Tage. So legt eine defizitorientierte Sicht, die im Alter(n) vorrangig Niedergang und Verlust wesentlicher menschlicher Eigenschaften und Fähigkeiten erblickt, eher die Berechtigung bzw. Notwendigkeit einer technischen Wiederherstellung früherer Niveaus körperlicher Funktions- und Leistungsfähigkeit nahe (Wigan 2013). Demnach müssten beispielsweise die physiologischen Standardwerte, die ein Monitoringsystem bei der Kontrolle von Puls, Blutdruck oder Zuckerspiegel zu Grunde legt, denen eines Menschen im mittleren Erwachsenenalter entsprechen (Izaks/Westendorp 2003). Auch die konzeptionelle Anlage technischer Mobilitätshilfen hätte idealerweise vergleichbare Freizeitaktivitäten oder sogar sportliche Leistungen zu ermöglichen wie in früheren Jahren (Loy 2020). Aus Sicht eines transhumanistischen Denkens, das die technische Verbesserung der ,natürlichen Grundausstattung des Menschen über das durch die Gesundheit definierte Normalmaß hinaus propagiert, müssten selbst diese Zielpunkte noch willkürlich und letzten Endes hinfällig erscheinen. Hier beginnen die Grenzen zwischen Rehabilitation und Enhancement, Wiederherstellung und Optimierung zu verschwimmen (Karpin/Mykitiuk 2008; s. Grunwald in diesem Band). Demgegenüber hätte eine Sichtweise, für die im Alter(n) eher die konstitutive Zerbrechlichkeit des Menschen als solchen zur Geltung kommt, durchaus Raum für körperliche Verletzlichkeits- und Unzulänglichkeitserfahrungen zu lassen. Die ,normale speziestypische Funktionsfähigkeit‘ (Boorse), auf die die technische Assistenz ausgerichtet ist, wäre gleichsam mit einem Altersgradienten zu versehen. Grundsätzlich ist in beiden Perspektiven zu beachten, dass sich das Verhältnis des Individuums zu seiner eigenen Körperlichkeit mit fortschreitendem Lebensalter verändert. Die 
für die spezifische ,Lebenslage“ des Menschen allgemein kennzeichnende „exzentrische Positionalität“ (Plessner 1928), der „,Abstand vom Körper im Körper““ (Fischer 2008, 597), wird im höheren Alter gleichsam akut. Der alternde Körper wird zunehmend in seiner unverfügbaren Materialität und Widerständigkeit erlebt und mag so irgendwann im wahrsten Sinne als ,Fremdkörper erscheinen (Coors 2020, 49-98). Entsprechend wird das Altern auch als ,Körperwerdung des Menschen' beschrieben, in der die prekäre physische Verfasstheit menschlichen Seins besonders radikal zu Tage tritt (Rentsch 2000). Mit Blick auf die Entwicklung im Bereich der Gerotechnologien gewinnt vor diesem Hintergrund die Frage nach den Auswirkungen technischer Assistenzsysteme auf das Verhältnis des alternden Menschen zu seinem eigenen Körper an Bedeutung und Brisanz. Das gilt insbesondere mit Blick auf invasivere Ansätze wie zum Beispiel die der Neuroprothetik, die tief in die spannungsvolle leib-körperliche Einheit menschlichen Seins eingreifen (Ihde 2008).

Die anthropologische Auseinandersetzung mit den Veränderungen menschlicher Körperlichkeit im Lebensverlauf lässt auch die grundlegende Bedeutung von Zeit und Zeitlichkeit für die Beschäftigung mit der Mensch-Technik-Interaktion im demographischen Wandel hervortreten. Das Defizitmodell des Alter(n)s setzt letztlich das mittlere Erwachsenenalter stillschweigend als eine Art menschlichen Normalzustand voraus, gegenüber dem das höhere Lebensalter gleichsam abfällt und als unvollkommenes und minderwertiges Verfallsstadium menschlichen Seins erscheint, dessen Unzulänglichkeiten mit technischen Mitteln so weit wie möglich auszugleichen sind. Tatsächlich sind die konstitutiven Mängel des ,Mängelwesens‘ Mensch (Gehlen 1986 [1940]) keineswegs allesamt zu jedem Zeitpunkt seiner Existenz gleichermaßen ausgeprägt. Auch sie haben jeweils ihre eigene Zeit. So hatte schon Gehlen das ,extrauterine Frühjahr (Portmann) als anthropologisches Charakteristikum betrachtet, weil die besondere entwicklungsbiologische Vulnerabilität und Hilflosigkeit des gleichsam unfertig geborenen Menschen zugleich seine spezifische Weltoffenheit und Kulturbedürftigkeit bedinge (ebd., 45). In loser Analogie wurde in der zeitgenössischen Gerontologie der Gedanke formuliert, im Alterungsprozess trete die „unvollendete Architektur der menschlichen Ontogenese“ zu Tage, die im höheren Lebensalter zunehmend Strategien der „Selektion, Optimierung und Kompensation“ (Baltes 1997) erforderlich mache. Blickt man allein auf den Aspekt der Adaptivität oder Funktionalität des Individuums, scheinen sich vor diesem Hintergrund zunächst in der Tat Entsprechungen zwischen früher Kindheit und höherem Lebensalter abzuzeichnen, wie sie im Topos des Alters als ,zweiter Kindheit‘ zum Ausdruck kommen (Covey 1993). Allerdings vernachlässigt eine derartige zeitindifferente Betrachtungsweise, dass die betreffenden Erscheinungen $\mathrm{zu}$ unterschiedlichen Punkten im Lebensverlauf des Individuums auftreten und auch entwicklungslo- 
gisch ganz anders perspektiviert sind. Auch die Entwicklung des alternden Menschen weist mithin unweigerlich nach vorn und lässt sich nicht angemessen als Regression in ein kindliches Stadium deuten. Dieser spezifische Zeithorizont des Alter(n)s hat auch Bedeutung für die Auseinandersetzung mit der Gerotechnologie. So droht eine zeitenthobene, vom lebensgeschichtlichen Zusammenhang abstrahierende Perspektive auf Funktionalität und ihre Einschränkungen nicht nur einer Infantilisierung älterer Menschen Vorschub zu leisten, wie sie etwa im Design gewisser Emotive oder Companion-Robots für ältere Menschen mit kognitiven Beeinträchtigungen zum Ausdruck kommt (Schweda/Jongsma 2018). Sie verkennt auch die konkrete lebensgeschichtliche Situiertheit und Ausrichtung der fraglichen Grenzsituationen, mit denen sich bis zuletzt spezifische Potenziale und Perspektiven menschlicher Entwicklung und persönlichen Wachstums auftun (Kruse 2017). Auf sie kann gerade eine dynamisch ,mitalternde‘ technische Assistenz nicht nur substitutiv oder kompensatorisch, sondern auch ermöglichend und fördernd bezogen sein (Nimrod 2020). Das gilt beispielsweise mit Blick auf die praktische Entfaltung der besonderen spirituellen Anliegen und Transzendenzbezüge hochaltriger Menschen (Kang et al. 2019).

Vergleichbar weitreichende Implikationen für die Betrachtung von Gerotechnologien ergeben sich schließlich auch mit Blick auf die anthropologische Bedeutung von Beziehungen und sozialer Verbundenheit im Horizont menschlichen Seins. So scheint dem Defizitmodell des Alter(n)s vielfach eine individualistische Anthropologie zu Grunde zu liegen, die den Menschen als ein wesentlich selbstständiges und unabhängiges Wesen begreift, das gleichsam sekundär und optional auch Beziehungen zu anderen aufnehmen und unterhalten kann. In diesem Licht erscheint das Alter(n) anthropologisch leicht als ein degenerativer Prozess bzw. ein defizitärer Zustand, der mit einem fortschreitenden Verlust an Selbständigkeit und einer wachsenden Angewiesenheit auf die Hilfe und Unterstützung anderer einhergeht. Assistive Technologien erhalten in der Perspektive eines solchen anthropologischen Individualismus von vornherein wie selbstverständlich die Bestimmung einer möglichst weitreichenden Wiederherstellung und Aufrechterhaltung individueller Selbständigkeit und Unabhängigkeit. Technische Assistenz dient vorrangig der Unterstützung von individuellen Fähigkeiten, die für eine eigenständige Lebensführung notwendig sind. Auf dieser Sichtweise fußt auch eine ideologische Lesart des Schlagworts vom ,Ageing in Place;, in der Technisierung letztlich ihre strategische Ausrichtung im Zusammenhang gesellschaftlicher Individualisierungsprozesse und ökonomischer Einsparpotenziale einer aktivierenden Sozial- und Alterspolitik erhält (Kenner 2008). Demgegenüber legen anthropologische Ansätze, die die ursprünglich relationale Natur des Menschen in den Vordergrund rücken und seine wesentliche Bezogenheit auf andere sowie die konstitutive Bedeutung sozialer Beziehungen für das mensch- 
liche Leben betonen, eine vollkommen andere Sicht des Alter(n)s nahe. Abhängigkeit und Angewiesenheit erscheinen nicht als defizitäre Modi des Menschseins, mit denen das alternde Individuum seinem näheren sozialen Umfeld oder einer sozialrechtlich unterstellten, Solidargemeinschaft" zur Last fällt, sondern als Ausdruck einer ursprünglichen menschlichen Bezogenheit und Verbundenheit (Rüegger 2020). Damit eröffnen sich auch andere Perspektiven auf die Bedeutung und Zielsetzung von Gerotechnologien. So wäre technische Assistenz etwa in den sozialen Zusammenhang eines Lebens in wechselseitigen Sorgebeziehungen einzubetten, die sich nicht dem Muster einer egoistisch motivierten strategischen Assoziation atomistischer Individuen fügen, sondern als Ausdruck einer anthropologisch konstitutiven Relationalität menschlichen Seins erscheinen. Eine praktische Konsequenz dieser Betrachtungsweise könnte in der Akzentuierung technologischer Ansätze bestehen, die zwischenmenschliche Hilfs- und Sorgebeziehungen ermöglichen oder unterstützen, etwa im Bereich der Kommunikations- oder Pflegetechnologien. Demgegenüber wäre eine mechanistische und funktionalistische Perspektive $\mathrm{zu}$ problematisieren, die die betreffenden Hilfsleistungen kurzerhand auf technisch substituierbare Assistenzfunktionen reduziert (Manzeschke 2019). Eine solche Sicht würde nicht nur die anspruchsvollen Voraussetzungen von Sorgeprozessen systematisch ausblenden, sondern alten Menschen und Pflegenden auch jeweils einseitig die Rolle von Empfängern bzw. Erbringern mechanischer Dienstleistungen zuweisen und so beide Seiten auf je andere Weise von der anthropologisch grundlegenden Erfahrung der Reziprozität menschlicher Sorgebeziehungen abschneiden (Assadi/Manzeschke/Kemmer 2020).

\section{Schluss}

Mit dem Verweis auf den Menschen, die Stufenfolge seines Lebens und den im höheren Alter zur Hilfe genommenen Stab hat Ödipus das Rätsel der Sphinx gelöst. Daraufhin stürzt sich das gefürchtete Ungeheuer von der Stadtmauer Thebens in den Tod. Die überlegene Kraft menschlicher Selbsterkenntnis hat die politische Gemeinschaft aus dem Bann der archaischen Mächte des Mythos zu Vernunft und Autonomie befreit.

Auch in dieser Hinsicht mag die kurze Geschichte zunächst sinnbildlich erscheinen für die Relevanz anthropologischer Fragestellungen in der Auseinandersetzung mit der Gerotechnologie. In der Tat erweist sich die grundsätzliche Frage nach der Bedeutung des Alter(n)s im Ganzen menschlichen Seins als maßgeblich für das Verständnis und die Bewertung technischer Assistenzsysteme für ältere Menschen. Dabei ist ihr insbesondere im Hinblick auf die körperliche 
Verfasstheit, zeitliche Ausrichtung sowie soziale Bezogenheit des Menschen weiter nachzugehen. Auf diese Weise werden Gesichtspunkte menschlicher Begrenztheit, Entwicklung und Relationalität erhellt, die auch weitreichende Implikationen für ethische und politische Diskussionen über die Mensch-TechnikInteraktion im demographischen Wandel haben.

Dabei kann es freilich keineswegs darum gehen, diese großen, grundsätzlichen Fragen nach der Natur des Menschen und den Bedingungen seiner Existenz kurzerhand abschließend zu beantworten, um vom Mythos überkommener Altersbilder geradewegs zum Logos einer wissenschaftlich fundierten Theorie über das Wesen des (alternden) Menschen fortzuschreiten. Vielmehr liegt die Bedeutung der anthropologischen Perspektive letztlich darin, einen begrifflich-theoretischen Rahmen und geistesgeschichtlichen Horizont für die systematische Analyse und Reflexion der in der Auseinandersetzung mit der Mensch-TechnikInteraktion im Alter(n) zu Grunde liegenden Vorstellungen menschlichen Seins zu eröffnen (ähnlich Schulz-Nieswandt 2018). Dabei hat sie allerdings zugleich in Rechnung zu stellen, dass das Aufkommen der Gerotechnologien auch seinerseits unser Verständnis und Erleben menschlichen Alter(n)s prägt und verändert. Auf diese Weise lässt sich schließlich vergegenwärtigen, was in der Auseinandersetzung letzten Endes im Spiel ist und auf dem Spiel steht: unser historisch gewachsenes und philosophisch ausbuchstabiertes Selbstverständnis als Menschen.

\section{Literatur}

Abdi, Jordan/Al-Hindawi, Ahmed/Ng, Tiffany/Vizcaychipi, Marcela P. (2018): Scoping review on the use of socially assistive robot technology in elderly care, in: BMJ Open 8/2, e018815.

Assadi, Galia/Manzeschke, Arne/Kemmer, Dominik (2020): Gutes Leben im Alter? Ethische und anthropologische Anmerkungen zu technischen Assistenzsystemen, in: Woopen, Christiane/Jahnsen, Anna/Mertz, Marcel/Genske, Anna (Hg.): Alternde Gesellschaft im Wandel. Zur Gestaltung einer Gesellschaft des langen Lebens, Berlin, 191-203.

Baltes, Margaret M./Wahl, Hans-Werner (1996): Patterns of communication in old age: The dependence-support and independence-ignore script, in: Health Communication 8/3, $217-231$.

Baltes, Paul B. (1997): Die unvollendete Architektur der menschlichen Ontogenese: Implikationen für die Zukunft des vierten Lebensalters, in: Psychologische Rundschau 48/4, 191-210.

Birkenstock, Eva (2008): Angst vor dem Altern? Zwischen Schicksal und Verantwortung, Freiburg i. Br./München.

Blackman, Stephanie/Matlo, Claudine/Bobrovitskiy, Charisse/Waldoch, Ashley/Fang, Mei Lan/Jackson, Piper/Mihailisdis, Alex/Nygård, Louise/Astell, Arlene/Sixsmith, Andrew 
(2016): Ambient assisted living technologies for aging well: A scoping review, in: Journal of Intelligent Systems 25/1, 55-69.

Byrne, Caroline A./Collier, Rem/O'Hare, Gregory M. P. (2018): A review and classification of assisted living systems, in: Information 9/7, 2078-2489.

Callahan Jr, James J. (Hg.) (2019): Aging in Place, Abingdon.

Cangelosi, Angelo/Invitto, Sara (2017): Human-robot interaction and neuroprosthetics: A review of new technologies, in: IEEE Consumer Electronics Magazine 6/3, 24-33.

Chaudhuri, Shomir/Thompson, Hilaire/Demiris, George (2001): Fall detection devices and their use with older adults: A systematic review, in: Journal of Geriatric Physical Therapy 37/4, 178-196.

Coors, Michael (2020): Altern und Lebenszeit. Phänomenologische und theologische Studien zu Anthropologie und Ethik des Alterns, Tübingen.

Covey, Herbert C. (1993): A return to infancy: Old age and the second childhood in history, in: The International Journal of Aging and Human Development 36/2, 81-90.

Dordoni, Paola/Argentero, Piergiorgio (2015): When age stereotypes are employment barriers: A conceptual analysis and a literature review on older workers stereotypes, in: Ageing International 40/4, 393-412.

Durick, Jeannette/Robertson, Toni/Brereton, Margot/Vetere, Frank/Nansen, Bjorn (2013): Dispelling ageing myths in technology design, in: Proceedings of the 25th Australian Computer-Human Interaction Conference, 467-476. https://doi.org/10.1145/2541016. 2541040.

Endter, Cordula (2016): Skripting age - the negotiation of age and aging in ambient assisted living, in: Domínguez-Rué, Emma/Nierling, Linda (Hg.): Ageing and Technology: Perspectives from the Social Sciences, Bielefeld, 121-140.

Fischer, Joachim (2008): Philosophische Anthropologie: Eine Denkrichtung des 20. Jahrhunderts, München.

Gehlen, Arnold (1986 [1940]): Der Mensch. Seine Natur und seine Stellung in der Welt, Wiesbaden.

Giese, Constanze: Überlegungen zum Einsatz der 'Pflegerobotik' und technischer Innovationen in der pflegerischen Versorgung, im vorliegenden Band.

Higgs, Paul/Gilleard, Chris (2014): Frailty, abjection and the 'othering' of the fourth age, in: Health Sociology Review 23/1, 10-19.

Höppner, Grit/Urban, Monika (2018): Where and how do aging processes take place in everyday life? Answers from a new materialist perspective, in: Frontiers in Sociology 3/7. doi: $10.3389 /$ fsoc.2018.00007.

Holm, Søren (2013): The implicit anthropology of bioethics and the problem of the aging person, in: Schermer, Maartje/Pinxten, Wim (Hg.): Ethics, Health Policy and (Anti-)aging: Mixed Blessings, Dordrecht, 59-71.

Ihde, Don (2008): Aging: I don’t want to be a cyborg!, in: Phenomenology and the Cognitive Sciences 7/3, 397-404.

Izaks, Gerbrand J./Westendorp, Rudi G. J. (2003): Ill or just old? Towards a conceptual framework of the relation between ageing and disease, in: BMC Geriatrics 3:7.

Jecker, Nancy S. (2020): Ending Midlife Bias: New Values for Old Age, Oxford.

Kaber, David B. (2018): Issues in human-automation interaction modeling: Presumptive aspects of frameworks of types and levels of automation, in: Journal of Cognitive Engineering and Decision Making 12/1, 7-24. 
Kang, Samantha L./Endacott, Camillee G./Gonzales, Gabrielle G./Bengtson, Vern L. (2019): Capitalizing and compensating: Older adults' religious and spiritual uses of technology, in: Anthropology and Aging 40/1, 14-31.

Karpin, Isabel/Mykitiuk, Roxanne (2008): Going out on a limb: Prosthetics, normalcy and disputing the therapy/enhancement distinction, in: Medical Law Review 16/3, 413-436.

Kenner, Alison Marie (2008): Securing the elderly body: Dementia, surveillance, and the politics of „aging in place“, in: Surveillance \& Society 5/3, 252-269.

Knowles, Bran/Hanson, Vicky L./Rogers, Yvonne/Piper, Anne Marie/Waycott, Jenny/Davies, Nigel (2019): $\mathrm{HCl}$ and aging: Beyond accessibility, in: Extended Abstracts of the $2019 \mathrm{CHI}$ Conference on Human Factors in Computing Systems. https://doi.org/10.1145/3290607. 3299025.

Kruse, Andreas (2017): Lebensphase hohes Alter: Verletzlichkeit und Reife, Berlin/Heidelberg. Künemund, H. (2016). Wovon hängt die Nutzung technischer Assistenzsysteme ab? Expertise zum Siebten Altenbericht der Bundesregierung, Berlin. https://nbn-resolving.org/urn:nbn: de:0168-ssoar-49994-1.

Lee, Hee Rin/Tan, Haodan/Šabanović, Selma (2016): That robot is not for me: Addressing stereotypes of aging in assistive robot design, in: 25th IEEE International Symposium on Robot and Human Interactive Communication (RO-MAN). IEEE, 2016, 312-317.

Loy, Jennifer (2020): Centenarian transhumanism aging in place, in: Biloria, Nimish (Hg.): Data-driven Multivalence in the Built Environment, Cham, 141-156.

Maalouf, Noel/Sidaoui, Abbas/Elhajj, Imad H./Asmar, Daniel (2018): Robotics in nursing: A scoping review, in: Journal of Nursing Scholarship 50/6, 590-600.

Madara Marasinghe, Keshini (2016): Assistive technologies in reducing caregiver burden among informal caregivers of older adults: A systematic review, in: Disability and Rehabilitation: Assistive Technology 11/5, 353-360.

Mannheim, Ittay/Schwartz, Ella/Xi, Wanyu/Buttigieg, Sandra C./McDonnell-Naughton, Mary/Wouters, Eveline J./Van Zaalen, Yvonne (2019): Inclusion of older adults in the research and design of digital technology, in: International Journal of Environmental Research and Public Health 16/19, 3718.

Manzeschke, Arne (2019): Roboter in der Pflege. Von Menschen, Maschinen und anderen hilfreichen Wesen, in: EthikJournal 5/1,1-11.

McNeill, Andrew/Coventry, Lynne (2015): Can we design stigma out of assistive walking technology?, in: First International Conference on Human Aspects of IT for the Aged Population 1.

Meyer, Sibylle/Mollenkopf, Heidrun (Hg.) (2010): AAL in der alternden Gesellschaft. Anforderungen, Akzeptanz und Perspektiven, Berlin/Offenbach.

Morris, Meg E./Adair, Brooke/Miller, Kimberly/Ozanne, Elizabeth/Hanson, Ralf/Pearce, Alan J./Santamaria, Nick/Viega, Luan/Long, Maureen/Said, Catherine M. (2013): Smart-home technologies to assist older people to live well at home, in: Journal of Aging Science 1/1, $1-9$.

Neven, Louis/Peine, Alexander (2017): From triple win to triple sin: How a problematic future discourse is shaping the way people age with technology, in: Societies 7/3, 26.

Nimrod, Galit (2020): Aging well in the digital age: Technology in processes of selective optimization with compensation, in: The Journals of Gerontology: Series B 75/9, 2008-2017. 
Parviainen, Jaana/Turja, Tuuli/van Aerschot, Lina (2018): Robots and human touch in care: Desirable and non-desirable robot assistance, in: Ge, Shuzhi Sam/Cabibihan, John-John/Salichs, Miguel A./Broadbent, Elizabeth/He, Hongsheng/Wagner, Alan R./Castro-González, Álvaro (Hg.): International Conference on Social Robotics, Cham, $533-540$.

Pirhonen, Jari/Tiilikainen, Elisa/Pekkarinen, Satu/Lemivaara, Marjut/Melkas, Helinä (2020): Can robots tackle late-life loneliness? Scanning of future opportunities and challenges in assisted living facilities, in: Futures 124, 102640.

Plessner, Hellmuth (1928): Die Stufen des Organischen und der Mensch, Berlin.

Pol, Margriet C./Poerbodipoero, Soemitro/Robben, Saskia/Daams, Joost/van Hartingsveldt, Margo/de Vos, Rien/de Rooij, Sophia E./Kröse, Ben/Buurman, Bianca M. (2013): Sensor monitoring to measure and support daily functioning for independently living older people: A systematic review and road map for further development, in: Journal of the American Geriatrics Society 61/12, 2219-2227.

Ray, Partha Pratim/Dash, Dinesh/De, Debashis (2019): A systematic review and implementation of loT-based pervasive sensor-enabled tracking system for dementia patients, in: Journal of Medical Systems 43/9, 287.

Rentsch, Thomas (2000): Altern als Werden zu sich selbst. Philosophische Anthropologie und Ethik der späten Lebenszeit, in: ders. (Hg.): Negativität und praktische Vernunft, Frankfurt a. M., 151-179.

Rossow, Judith (2012): Einführung: Individuelle und kulturelle Altersbilder, in: Berner, Frank/Rossow, Judith/Schwitzer, Klaus-Peter (Hg.): Individuelle und kulturelle Altersbilder, Wiesbaden, 9-24.

Rüegger, Heinz (2020): Beyond control. Dependence and passivity in old age, in: Schweda, Mark/Coors, Michael/Bozzaro, Claudia (Hg.): Aging and Human Nature: Perspectives from Philosophical, Theological and Historical Anthropology, Cham, 47-57.

Schicktanz, Silke/Schweda, Mark (2021): Aging 4.0? - Rethinking the ethical framing of technology-assisted eldercare, in: History and Philosophy of the Life Sciences (forthcoming).

Schmidt, Laura/Wahl, Hans-Werner (2019): Alter und Technik, in: Hank, Karsten/Schulz-Nieswandt, Frank/Wagner, Michael/Zank, Susanne (Hg.): Alternsforschung, Berlin, 537-556.

Schulz, Richard/Wahl, Hans-Werner/Matthews, Judith T./De Vito Dabbs, Annette/Beach, Scott R./Czaja, Sara J. (2015): Advancing the aging and technology agenda in gerontology, in: The Gerontologist 55/5, 724-734.

Schulz-Nieswandt, Frank (2018): Zur Metaphysikbedürftigkeit der empirischen Alter(n) ssozialforschung, Berlin.

Schwab, Gustav (1986): Sagen des klassischen Altertums, Frankfurt a.M.

Schweda, Mark (2018): Alter(n) in Philosophie und Ethik, in: Schroeter, Klaus/Vogel, Claudia/Künemund, Harald (Hg.): Handbuch Soziologie des Alter(n)s, Wiesbaden. https://doi.org/10.1007/978-3-658-09630-4_3-1.

Schweda, Mark/Coors, Michael/Bozzaro, Claudia (2020): Introduction, in: Schweda, Mark/Coors, Michael/Bozzaro, Claudia (Hg.): Aging and Human Nature: Perspectives from Philosophical, Theological and Historical Anthropology, Cham, 1-9. 
Schweda, Mark/Jongsma, Karin (2018): ,Rückkehr in die Kindheit'oder ,Tod bei lebendigem Leib'? Ethische Aspekte der Altersdemenz in der Perspektive des Lebensverlaufs, in: Zeitschrift für Praktische Philosophie 5/1, 181-206.

Sharkey, Noel/Sharkey, Amanda (2010): The crying shame of robot nannies: An ethical appraisal, in: Interaction Studies 11/2, 161-190.

Stavropoulos Thanos G./Papastergiou, Asterios/Mpaltadoros, Lampros/Nikolopoulos, Spiros/Kompatsiaris, loannis (2020): loT wearable sensors and devices in elderly care: A literature review, in: Sensors 20/10, 2826.

Trappe, Tobias (2002): Vom Alter. Vorüberlegungen zur Substanzanthropologie (II), in: Phänomenologische Forschungen, 109-130.

Van Dyk, Silke/Lessenich, Stephan (Hg.) (2009): Die jungen Alten. Analysen einer neuen Sozialfigur, Frankfurt a.M.

Vines, John/Pritchard, Gary/Wright, Peter/Olivier, Patrick/Brittain, Katie (2015): An age-old problem: Examining the discourses of ageing in $\mathrm{HCl}$ and strategies for future research, in: ACM Transactions on Computer-Human Interaction (TOCHI) 22/1, 1- 27.

Wahl, Hans-Werner (2016): Gero-Technologie. Hintergrundpapier zum Positionspapier der Deutschen Gesellschaft für Gerontologie und Geriatrie DGGG e.V. https://www.dgggonline.de/fileadmin/user_upload/201607_Hintergrundpapier_Positionspapier_Alter-undTechnik_DGGG.pdf.

Wanka, Anna/Gallistl, Vera (2018): Doing age in a digitized world - A material praxeology of aging with technology, in: Frontiers in Sociology 3, 6.

Westberg, Kate/Reid, Mike/Kopanidis, Foula (2020): Age identity, stereotypes and older consumers' service experiences, in: Journal of Services Marketing https://doi.org/10. 1108/JSM-10-2019-0386.

Wigan, Marcus (2013): Constructing age and technology as augmentation, not degradation: Exploring what the aged themselves think they need, not what is decided for them, in: 2013 IEEE International Symposium on Technology and Society (ISTAS): Social Implications of Wearable Computing and Augmediated Reality in Everyday Life, 136-143.

Wurm, Susanne (2020): Altersbilder und Gesundheit. Grundlagen - Implikationen Wechselbeziehungen, in: Frewer, Andreas/Klotz, Sabine/Herrler, Christoph/Bielefeldt, Heiner (Hg.): Gute Behandlung im Alter? Menschenrechte und Ethik zwischen Ideal und Realität, Bielefeld, 25-42. 